\title{
Gap Analysis between Perceptions and Expectations of Medical-Surgical Patients in a Public Hospital in Saudi Arabia
}

\author{
Mohammed Mahmoud Al-Momani \\ Department of Community Health Sciences, College of Applied Medical Sciences, King Saud University, \\ Riyadh, Kingdom of Saudi Arabia
}

\section{Key Words}

Nursing care - Patient perception - Patient expectation .

Patient satisfaction - Quality of nursing care

\begin{abstract}
Objectives: To investigate patients' satisfaction with nursing care by measuring the gap between patients' expectations of care and perceptions of the actual care provided and to identify the areas of nursing care that need improvement. Subjects and Methods: A cross-sectional survey was conducted among patients who were admitted to the Departments of Medicine and Surgery at King Saud Medical City, Riyadh, Saudi Arabia. A modified Service Quality (SERVQUAL) instrument was adapted to collect information from a convenience sample of 432 patients from November 25, 2012, to February 3, 2013. The instrument comprised 22 pairs of questions assessing 5 dimensions of the nursing care provided to patients during hospitalizations. The mean patient expectations and perceptions as well as the gap score values for each dimension of nursing service were tested for differences between the mean scores of the sample at a level of significance of 0.05 using a t test. Results: The gap score for all of the 5 dimensions of nursing services were: responsiveness, -1.71 ; reliability, -1.48 ; tangibles, -1.36 ; assurance,
\end{abstract}

-1.26 , and empathy, -0.96 . Service quality across the dimensions of responsiveness and reliability was statistically significant $(p<0.05)$. This result indicated that patients were not satisfied with the nursing service quality in relation to all dimensions. Conclusion: Our study showed negative gaps for the 5 nursing service quality dimensions evaluated. This could provide nurses with information about the aspects of nursing care that promote more positive patient outcomes and satisfaction.

(c) 2015 S. Karger AG, Basel

\section{Introduction}

Patient satisfaction is a critical component in determining the quality of health care services, and it is a major component of the philosophy of patient-centred care [1, 2]. Service quality largely determines patient satisfaction [3-5]. A popular and widely used definition of service quality is conformance to the patient's expectations $[1,6$, 7], and that serves as a reference point in patients' assessment of health care performance [6].

Several tools have been developed for measuring patient satisfaction regarding patients' perceptions and expectations. These tools vary in their definitions, content,

\section{KARGER}

E-Mail karger@karger.com www.karger.com/mpp
This is an Open Access article licensed under the terms of the Creative Commons Attribution-NonCommercial 3.0 Unported license (CC BY-NC) (www.karger.com/OA-license), applicable to the online version of the article only. Distribution permitted for non-commercial purposes only.
Mohammed Mahmoud Al-Momani

Department of Community Health Sciences

College of Applied Medical Sciences, King Saud University

King Abdullah Street, PO Box 10219, Riyadh 11433 (Kingdom of Saudi Arabia)

E-Mail malmomani@ksu.edu.sa 
and measurement [8]. Therefore, most measures of patient satisfaction in nursing care have failed to capture key nursing activities and are therefore poor indicators of nursing care [9]. However, the Service Quality (SERVQUAL) scale developed by Parasuraman et al. [10] is largely responsible for addressing these deficiencies and remains the most widely used tool [11] for measuring patient satisfaction and service quality $[12,13]$. The SERVQUAL scale has been modified and used in a wide array of studies in nursing care to assess patient satisfaction in a number of service categories to suite some study purposes $[8,11,14,15]$. Most of the studies conducted in Saudi Arabian hospitals have focused on assessing the quality of nursing services by measuring only patient perceptions [16-19]. The SERVQUAL scale examines the gap between patients' expectations and perceptions. Hence, in this study, SERVQUAL was used to investigate patients' satisfaction with nursing care by measuring the gap between patients' expectations of care and the perceived nursing care actually delivered and to identify the areas of nursing care that need improvement.

\section{Subjects and Methods}

This cross-sectional, descriptive survey was conducted with patients in medical and surgical wards of a 1,500-bed hospital at King Saud Medical City in Riyadh, Saudi Arabia. A convenience sample of 432 adult patients who were discharged from the hospital was surveyed. The inclusion criteria were: age 18 years or older, able to read and understand Arabic, not suffering from severe mental or cognitive disorders, able to coherently communicate and conscious, hospital stay longer than 3 nights, willing to complete a questionnaire and healthy enough to complete the survey. This study was conducted from November 25, 2012, to February 3, 2013.

In this study the SERVQUAL scale was adapted to be applicable to the service quality expected in Saudi Arabian hospitals, where the health care system is different from those of the USA and European countries. A focus group discussion was conducted with 18 males and 13 females from the study group prior to initiating this study to collect meaningful data on patient care and to generate items for the questionnaire. The emphasis was on the list of questions developed from the common themes that represented the most significant areas for determining patients' expectations and perceptions in the medical-surgical wards. The questionnaire was divided into 2 parts: the first part collected demographic data and the other collected information about patients' expectations and perceptions of nursing care. The scale items were translated from English to Arabic and back-translated firstly by the author. The translation was also verified by specialists to ensure the reliability of the scale. The instrument comprised 22 pairs of questions comparing patients' expectations to their perceptions in 5 dimensions as follows: (a) tangibles: the appearance of the physical facilities, equipment, personnel, and communication materials; (b) reliability: the ability to perform the promised nursing care dependably and accurately; (c) responsiveness: the willingness to help patients and to provide prompt nursing care; (d) assurance: the knowledge and courtesy of nurses and their ability to convey trust and confidence, and (e) empathy: the provision of caring, individualized attention to customers, including access, communication, and understanding.

Highly educated selected staff members of the College of Nursing investigated the content validity, appropriateness, readability, and completion of the questionnaire items. The questionnaire was then modified by incorporating the experts' comments. The internal consistency of the survey instrument was tested using Cronbach's a (0.73). Stability/reliability was tested using test-retest procedures. The modified questionnaire was piloted in 10 male and 10 female patients from medical and surgical wards who were not included in this study. They were asked to complete the questionnaire and comment on its structure, the clarity of the items, and the ease of completion.

Two final-year nursing students collected data from eligible patients after obtaining verbal consent. Anonymity and confidentiality were ensured. The questionnaire was completed by patients while they were in comfortable conditions and able to communicate, so that their responses were as independent and accurate as possible.

Each questionnaire took approximately $20-30 \mathrm{~min}$ to complete. Five hundred questionnaires were distributed. Patients rated their expectations and perceptions of the care provided on a 5-point scale as follows: highest, 5; high, 4; moderate, 3; low, 2, and lowest, 1 . The responses were scored, and the levels of expectation and perception were calculated.

Permission to conduct this study was obtained from the Institution Committee on Research, while permission to gather the data was obtained from the nursing director of the hospital. The procedure was explained to all of the participants prior to the interview. All of the data obtained in this study were handled confidentially.

\section{Statistical Analysis}

The data were coded, entered in to a computer, and analysed using Statistical Package for Social Sciences (SPSS) version 18 (USA). The scores associated with nursing care in each dimension and the overall perception scores were calculated. A descriptive analysis with frequencies and percentages was generated to describe patients' demographic profiles. The mean score of patients' expectations and perceptions and the gap scores of the 22 items were calculated. The mean patient expectation and perception values for each dimension of nursing services were tested for differences between the mean scores of the sample at a level of significance of 0.05 using a t test. Patient satisfaction and nursing service quality were determined by the difference between the mean scores of expected and perceived care for each item. If the perception exceeded the expectation, the service quality was considered highly satisfactory. If the perception equalled the expectation, the service quality was considered satisfactory. However, if the expectation exceeded the perception, the service quality was considered below satisfactory $[13,14]$. The relationship between patient satisfaction mean scores and their demographic attributes was analysed using a t test and ANOVA; $\mathrm{p}<0.05$ was considered statistically significant.
Al-Momani 


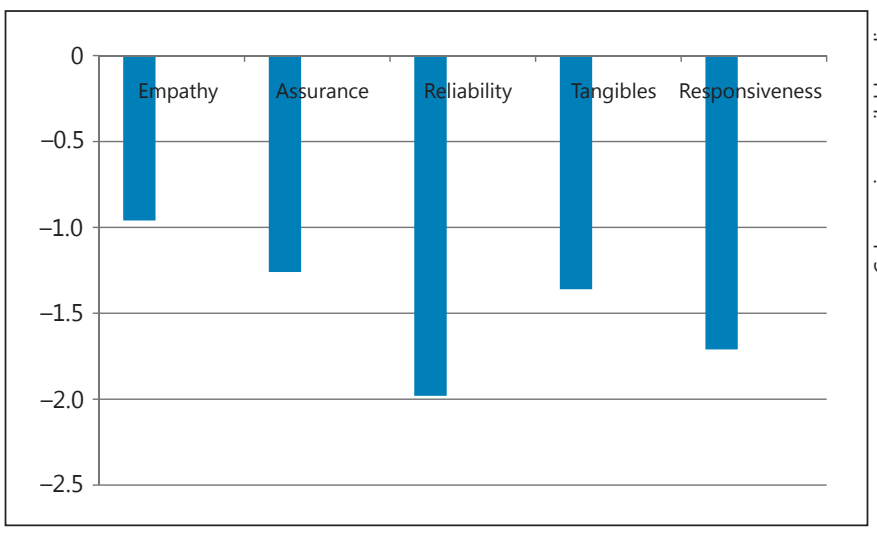

Fig. 1. Gap scores for each dimension of hospital nursing care.

\section{Results}

The findings of the pilot test indicated that the instrument was clear, understandable, and representative of their needs.

Of the 500 questionnaires distributed, 432 (86.4\%) were completed. The mean age of the participants was $48.7 \pm 6.5$ years (range $21-69$ ). Of the 432 participants, $284(65.7 \%)$ were married and $326(75.5 \%)$ had a school level education. The range of length of stay in the hospital was 3-16 days, and most patients $(\mathrm{n}=164 ; 38 \%)$ had stayed longer than 10 days. Of the 432 participants, 290 (67.1\%) were admitted to surgical departments (table 1).

The mean expectation scores for individual statements ranged from the highest mean score of $4.94 \pm 0.61$ for 'nurses respect patient values, beliefs and culture' to the lowest mean score of $4.48 \pm 0.51$ for 'nurses provide nursing care as they have promised' (table 2).

The mean perception scores for individual statements ranged from the highest mean score of $4.82 \pm 1.07$ for 'nurses wear clean and neat uniforms' to the lowest mean score of $1.85 \pm 0.96$ for 'nurses provide informative brochures about hospital policies' (table 3 ).

The service quality gap scores (i.e. the discrepancy between patients' expectations and perceptions) where negative gaps occurred in the 5 dimensions indicated that patients' expectations generally were not being met. The largest gap was -1.71 for responsiveness, followed by -1.48 for reliability, -1.36 for tangibles, -1.26 for assurance and -0.96 for empathy, in that order, as indicated in fig 1.

The statements of nursing care that scored the largest mean differences between expectations and perceptions
Table 1. Demographic characteristics of the respondents $(n=432)$

\begin{tabular}{lr}
\hline Variable & $\mathrm{n}(\%)$ \\
\hline Gender & \\
$\quad$ Male & $244(56.5)$ \\
Female & $188(43.5)$ \\
Age & $74(17.1)$ \\
$\quad \leq 20$ years & $184(42.6)$ \\
$21-40$ years & $110(25.5)$ \\
$41-60$ years & $64(14.8)$ \\
60+ years & \\
Marital status & $148(34.3)$ \\
Single & $284(65.7)$ \\
Married & \\
Educational level & $326(75.5)$ \\
$\quad$ Primary and secondary & $106(24.5)$ \\
Postsecondary & \\
Length of stay & $156(36.1)$ \\
3-6 days & $112(25.9)$ \\
$7-10$ days & $164(38.0)$ \\
10+ days & $126(32.9)$ \\
Department & $290(67.1)$ \\
Medical &
\end{tabular}

were 'nurses provide informative brochures about hospital policies' $(-2.69)$ with $\mathrm{p}=0.037$, followed by 'nurses provided the patient with adequate and accurate information about his/her condition' (-2.61) with $\mathrm{p}=0.041$. The smallest gap scores, which represented the smallest difference between patient expectations and perceptions, were for the statement 'nurses wear clean and neat uniforms' (-0.08), followed by 'nurses respect the patient's values, beliefs and culture' $(-0.23)$ (table 4$)$.

The gap scores for demographic characteristics are displayed in table 5, and there was no significant relationship between gender, age, marital status, length of stay, and type of department ( $p>0.05)$. However, a significant relationship was found between level of education and overall gap score $(\mathrm{p}<0.05)$. Those who had a postsecondary school education (1.65) had higher gap scores than those with a primary and secondary school education $(-1.15)$.

\section{Discussion}

The results of this study showed that the overall expectation scores for all of the SERVQUAL dimensions (responsiveness, reliability, tangibles, assurance and em- 
Table 2. The 5 highest and lowest patient expectation mean scores $(n=432)$

\begin{tabular}{lr}
\hline Aspects of care & Mean \pm SD \\
\hline Highest patient expectation mean scores & $4.94 \pm 0.61$ \\
Nurses respect the patient's values, beliefs and culture & $4.88 \pm 0.59$ \\
Nurses help the patient feel safe and confident when receiving nursing care & $4.87 \pm 0.64$ \\
Nurses show competence in solving the patient's problems & $4.86 \pm 0.49$ \\
Nurses are able to understand the patient's inquiries and communicate with the patient in Arabic & $4.86 \pm 0.68$ \\
Nurses provided the patient with adequate and accurate information about his/her condition & $4.48 \pm 0.51$ \\
\hline Lowest patient expectation mean scores & $4.54 \pm 0.61$ \\
Nurses provide nursing care as they have promised & $4.55 \pm 0.69$ \\
Nurses provide informative brochures about hospital policies & $4.62 \pm 0.57$ \\
Nurses maintain the patient's privacy and confidentiality & $4.64 \pm 0.71$ \\
Nurses always respond to phone calls from the patient's family & \\
Nurses always seem genuinely concerned about patient's pain, fear or unhappiness & \\
\hline
\end{tabular}

Table 3. The 5 highest and lowest patient perception mean scores $(n=432)$

\begin{tabular}{lr} 
Aspect of care & Mean \pm SD \\
\hline Highest perception mean scores & $4.82 \pm 1.07$ \\
Nurses wear clean and neat appearing uniforms & $4.71 \pm 0.56$ \\
Nurses respect the patient's values, beliefs and culture & $4.17 \pm 0.71$ \\
Nurses are willing to help the patient at all times & $4.12 \pm 0.65$ \\
Nurses talk with the patient politely and have a pleasant tone of voice & $4.11 \pm 0.61$ \\
Nurses make the patient feel safe and confident when receiving nursing care at the hospital & $1.85 \pm 0.96$ \\
\hline Lowest perception mean scores & $2.21 \pm 0.87$ \\
Nurses provide informative brochures about hospital policies & $2.25 \pm 1.08$ \\
Nurses give a clear explanation before providing any nursing care (e.g. i.v. fluid) & $2.33 \pm 1.13$ \\
Nurses provided the patient with adequate and accurate information about his/her condition & $2.81 \pm 0.89$ \\
Nurses always respond to phone calls from the patient's family & Nurses are always available to answer the patient's questions
\end{tabular}

pathy) and for all of the individual statements of nursing care were higher than the corresponding perception scores, resulting in negative SERVQUAL gap scores. This finding was confirmed by the results of the study of Uzun [8], in which negative results were found in all 5 dimensions of nursing services. These gap scores indicated that these areas need improvement, and the study population felt strongly about their needs and choices, specifically for 'responsiveness' and 'reliability' items of nursing services.

The statement 'nurses provide informative brochures about hospital policies', included in the tangible dimension, had the largest mean gap score. This finding is congruent with the findings of a study conducted in Northern Greece that also showed that lack of informative brochures had the largest mean gap scores because of high expectation scores [15]. The congruency of both studies could be due to the fact that patients use this service to orient themselves in relation to hospital policies, which helps them address their needs.

The findings revealed that the study population had a large gap score for nursing care regarding the information they were given about their condition and the explanations given prior to initiating nursing care. This result could be interesting because most of the nurses working at this hospital are non-native, which diminishes the role they play in health education and their ability to maintain good communication with patients [16]. A study reported that when nurses communicate well with their patients and give them honest and appropriate information with clear explanations, patients have less stress and fewer complaints [20]. 
Table 4. The 5 largest and smallest gap scores for each aspect of nursing care $(n=432)$

\begin{tabular}{lc}
\hline Aspect of nursing care & Service gap score (P-E) \\
\hline The 5 largest gap scores & -2.69 \\
Nurses provide informative brochures about hospital policies & -2.61 \\
Nurses provide the patient with adequate and accurate information about his/her condition & -2.47 \\
Nurses give a clear explanation before providing any nursing care (e.g. i.v. fluid) & -2.29 \\
Nurses always respond to phone calls from the patient's family & -1.97 \\
Nurses are always available to answer the patient's questions & 0.037 \\
\hline The 5 smallest gap scores & -0.76 \\
Nurses maintain the patient's privacy and confidentiality & -0.67 \\
Nurses are willing to help the patient at all times & -0.64 \\
Nurses talk with the patient politely and with a pleasant tone of voice & -0.23 \\
Nurses respect the patient's values, beliefs and culture & -0.08 \\
Nurses always wear clean and neat uniforms & 0.046 \\
\hline P = Perception; E $=$ expectation. & 0.054 \\
\hline
\end{tabular}

Two statements ('nurses always respond to phone calls from the patient's family' and 'nurses are always available to answer the patient's questions') from the responsiveness dimension had high gap scores because of high patient expectations. A probable explanation could be the high nurse-to-patient ratio in the hospital, which was 1:8 in morning shift but decreased to 1:13 in the night shift and during the Hajj season, as found in most public hospitals that provide unpaid health services to patients [16]. The patient-to-nurse ratio is significantly associated with patients' satisfaction levels and their satisfaction regarding the quality of nursing care [20].

The smallest gap scores were distributed relatively equally among the 5 dimensions (responsiveness, reliability, tangibles, assurance and empathy). Notably, the empathy dimension obtained 2 of the 5 smallest gap scores. These statements were related to respecting the patient's values, beliefs and culture, as well as the perceived level of politeness and pleasantness when nurses spoke with patients. These 2 aspects are very important to patients, who always rank them as most important in their expectations.

The findings of this study revealed no significant relationships between gender, age, marital status, length of stay or type of department and the overall gap scores. However, a significant relationship was found with level of education. Patients with a high-school education had higher gap scores. This result indicated that they were dissatisfied with the nursing care that was provided, probably because these patients had higher expectations prior to admission and were more knowledgeable about their
Table 5. Service gap scores (P-E) of the respondents' demographic characteristics $(n=432)$

\begin{tabular}{lllll}
\hline Variable & $\begin{array}{l}\mathrm{E} \\
\text { mean } \\
\text { score }\end{array}$ & $\begin{array}{l}\mathrm{P} \\
\text { mean } \\
\text { score }\end{array}$ & $\begin{array}{l}\text { Service } \\
\text { gap } \\
\text { score } \\
(\mathrm{P}-\mathrm{E})\end{array}$ & $\begin{array}{l}\mathrm{p} \\
\text { value }\end{array}$ \\
& & & & \\
& & & & \\
\hline Gender & 4.78 & 3.41 & -1.37 & 0.62 \\
$\quad$ Male & 4.70 & 3.37 & -1.33 & \\
$\quad$ Female & & & & \\
Age & 4.71 & 3.42 & -1.29 & 0.38 \\
$\quad$ <20 years & 4.75 & 3.40 & -1.35 & \\
$\quad$ 21-40 years & 4.80 & 3.36 & -1.44 & \\
$\quad$ 41-60 years & 4.68 & 3.38 & -1.30 & \\
$\quad$ 60+ years & & & & \\
Marital status & 4.72 & 3.44 & -1.28 & 0.50 \\
$\quad$ Single & 4.76 & 3.34 & -1.42 & \\
$\quad$ Married & & & & \\
\hline Educational level & 4.51 & 3.36 & -1.15 & 0.01 \\
$\quad$ Primary and secondary & 4.89 & 3.24 & -1.65 & \\
$\quad$ Postsecondary & & & & \\
Length of stay & 4.70 & 3.38 & -1.42 & 0.07 \\
$\quad$ 3-6 days & 4.74 & 3.37 & -1.37 & \\
$\quad$ 7-10 days & 4.78 & 3.42 & -1.36 & \\
$\quad$ 10+ days & & & & \\
Department & 4.67 & 3.28 & -1.39 & 0.06 \\
$\quad$ Medical & 4.81 & 3.50 & -1.31 & \\
$\quad$ Surgical & & & & \\
\hline
\end{tabular}

$\mathrm{P}=$ Perception; $\mathrm{E}=$ expectation . 
needs and rights, resulting in a more critical evaluation of their experiences with nursing care [19].

The limitations of this study were: the convenience sampling, which affected the ability to generalize the findings, and the lack of consideration of the past experience of those admitted to the hospital or the condition for which the patients were admitted, because these variables could have influenced the patients' expectations and perceptions of nursing care. Additionally, this study was not correlated to the outcome of treatment (either medical or surgical).

\section{Conclusion}

The findings of this study showed that the scores for patient expectations regarding nursing care exceeded the scores for perception of the actual care provided in all dimensions of responsiveness and reliability items of nursing services. These gap scores identified many areas that require improvement. This study provides nurses with information about the aspects of nursing care that could enhance patient satisfaction, such as responding to their needs promptly, providing patients with adequate infor- mation about their condition, respecting patients' families and responding to their phone calls, and providing patients with informative brochures about hospital policies.

Further studies sampling consecutive patients across multiple public hospitals and/or a comparative study between public hospitals and other types of hospitals might be needed to evaluate the impact of particular factors on the quality of nursing services.

\section{Acknowledgement}

The author would like to extend his appreciation to the College of Applied Medical Sciences Research Centre and the Deanship of Scientific Research at King Saud University for funding this research (CAMS-RC3334/26). The author would like to thank the administration of King Saud Medical City for their cooperation and for giving their permission for successful data collection. The contribution of Rihab Al Ali and Hamad Qahttani towards data collection for this study is acknowledged. Special thanks go to Dr. Baderldin Mohammed for statistical consultations in each step of this study. Thanks also go to Dr. Ghalib Rababah and Dr. Na'el Shara'h for their help in verifying the translations. Appreciation and thanks go to all who helped investigate the content validity of the data collection tool.

\section{References}

1 Baba I: Experiences in quality assurance at Bawku Hospital Eye Department, Ghana. Community Eye Health 2004;17:31-35.

2 Palese A, et al: Surgical patient satisfaction as an outcome of nurses' caring behaviors: a descriptive and correlational study in six European countries. J Nurs Scholarsh 2011;43: 341-350.

- 3 Reck DL: Can and should nurses be aware of patients' satisfaction and quality of nursing care. Nurs Adm Q 2013;53:224-230.

4 Calnan M: Towards a conceptual framework of lay evaluation of health care. Soc Sci Med 1988;27:927-933.

5 Roter DL, Hall JA, Katz NR: Relations between physicians, behaviors and analogue patients' satisfaction, recall, and impressions. Med Care 1987;25:437-451

6 Boxer B, Goldfarb E: The expectations and perceptions of ED patients. J Emerg Nurs 2009;35:540-541.

7 Mrayyan MT: Jordanian nurses' job satisfaction, patients' satisfaction and quality of nursing care. Int Nurs Rev 2006;53:224-230.
-8 Uzun O: Patient satisfaction with nursing care at a university hospital in Turkey. J Nurs Care Qual 2001;16:24-33.

9 Chang K: Dimensions and indicators of patients' perceived nursing care quality in the hospital setting. J Nurs Care Qual 1997;11: 26-37.

10 Parasuraman A, Zeithaml VA, Berry LL: A conceptual model of service quality and its implications for future research. J Mark 1985; 49:41-50.

11 Peprah AA, Atarah BA: Assessing patients satisfaction using SERVQUAL model: a case of Sunyani Regional Hospital, Ghana. Int J Bus Soc Res 2014;4:133-143.

12 Tomlinson JS, Ko CY: Patient satisfaction: an increasingly important measure of quality. Ann Surg Oncol 2006;13:764-765

13 Babakus E, Mangold WG: Adapting the SERVQUAL scale to hospital service: an empirical investigation. Health Serv Res 1992;26: 777-786.

14 Scardina SA: SERVQUAL: a tool for evaluating patient satisfaction with nursing care. J Nurs Care Qual 1994;8:38-46.
15 Karassavidou E, Glavel N, Papadopoulos CT: Quality in NHS hospitals: no one knows better than patients. Meas Bus Excell 2009;13: 34-46.

16 Gilay A, Al-Wehady A: Job satisfaction of female Saudi nurses. East Mediterr Health J 2001;7:31-37.

17 Halligan P: Caring for patients of Islamic denomination: critical care nurses' experiences in Saudi Arabia. J Clin Nurs 2006;15:15651573.

18 Suliman WA, Welmann E, Omar I, et al: Applying Watson's nursing theory to assess patient perceptions of being cared for in a multicultural environment. J Nurs Res 2009; 17: 293-300.

19 Al Momani M, Al Korashy H: Patient experience of nursing quality in a teaching hospital in Saudi Arabia. Iran J Public Health 2012;41: $1-8$.

20 Radwin L, et al: Cancer patients' description of their nursing care. J Adv Nurs 2005;50: 162-169. 